\title{
NUMERICAL EXPERIMENT WITH UPSTREAM BOUNDARY CONDITIONS
}

\begin{abstract}
Jaroslav HUML, Karel KOZEL *
Abstract: The work deals with a numerical solution of subsonic and transonic flows described by the system of Euler equations in $2 D$ and $3 D$ flows in a channel. Authors used Lax-Wendroff and the multistage Runge-Kutta scheme to numerically solve the flows in a 2DGAMM channel and an extended 3D channel. Authors compare the results achieved by two different upstream boundary conditions in 2D and also in 3D transonic channel flows.
\end{abstract}

\section{INTRODUCTION}

To simulate a flow of an inviscid (compressible) fluid, according to a 1D analysis we should prescribe such boundary conditions on the inlet and outlet which depend on the magnitude of a normal component of velocity. If a normal component of velocity is higher than a local speed of sound, it is necessary to consider all the variables on the inlet and on the outlet no one. In the opposite case, 3 (or 4 in a $3 \mathrm{D}$ case) variables are prescribed on the inlet and the others are extrapolated from the flow field or computed. What influence on the numerical solution do the upstream boundary conditions have? Or can we prescribe all the variables on the inlet for a normal component of velocity smaller than a local speed of sound? That were questions which we tried to find out.

\section{Mathematical models}

The two-dimensional flow of an inviscid compressible fluid is described by the system of Euler equations.

$$
W_{t}+F_{x}+G_{y}=0
$$

where

$$
\begin{gathered}
W=(\rho, \rho u, \rho v, e)^{T}, \\
F=\left(\rho u, \rho u^{2}+p, \rho u v,(e+p) u\right)^{T}, G=\left(\rho v, \rho u v, \rho v^{2}+p,(e+p) v\right) \\
p=(\kappa-1)\left[e-\frac{1}{2} \rho\left(u^{2}+v^{2}\right)\right]
\end{gathered}
$$

and for 3D case of an inviscid flow

$$
W_{t}+F_{x}+G_{y}+H_{z}=0
$$

where

$$
\begin{gathered}
W=(\rho, \rho u, \rho v, \rho w, e)^{T}, \\
F=\left(\rho u, \rho u^{2}+p, \rho u v, \rho u w,(e+p) u\right)^{T}, G=\left(\rho v, \rho u v, \rho v^{2}+p, \rho v w,(e+p) v\right)^{T}, \\
H=\left(\rho w, \rho u w, \rho v w, \rho w^{2}+p,(e+p) w\right)^{T} \\
p=(\kappa-1)\left[e-\frac{1}{2} \rho\left(u^{2}+v^{2}+w^{2}\right)\right]
\end{gathered}
$$

* Jaroslav HUML, Czech Technical University in Prague, Faculty of Mechanical Engineering, Karlovo náměstí 13, CZ-121 35 Prague 2, jar.huml@centrum.cz.

Karel KOZEL, Czech Technical University in Prague, Faculty of Mechanical Engineering, Karlovo náměstí 13, CZ-121 35 Prague 2, karel.kozel@fs.cvut.cz.

This is an Open Access article distributed under the terms of the Creative Commons Attribution License 2.0, which permits unrestricted use, distribution, and reproduction in any medium, provided the original work is properly cited. 
In the above equations, $\rho$ denotes density, $u, v, w$ are components of velocity in the direction of axis $x, y, z, p$ is pressure, $e$ is total energy per a unit volume. The parameter $\kappa=1.4$ is the adiabatic exponent.

\section{NUMERICAL METHODS}

For modeling of the mentioned flow cases, two numerical schemes of the finite volume method were used on non-orthogonal structured grids of quadrilateral $D_{i j}$ or hexahedral cells $D_{i j k}$.

- the Lax-Wendroff scheme - Richtmyer form (LW)

predictor step

$$
W_{i, j}^{n+1 / 2}=W_{i, j}^{n}-\frac{\Delta t}{2 \mu_{i, j}} \sum_{k=1}^{4}\left(\tilde{F}_{k}^{n} \Delta y_{k}-\tilde{G}_{k}^{n} \Delta x_{k}\right)
$$

corrector step

$$
W_{i, j}^{n+1}=W_{i, j}^{n}-\frac{\Delta t}{\mu_{i, j}} \sum_{k=1}^{4}\left(\tilde{F}_{k}^{n+1 / 2} \Delta y_{k}-\tilde{G}_{k}^{n+1 / 2} \Delta x_{k}\right)+A D\left(W_{i, j}^{n}\right)
$$

- the multistage Runge-Kutta method (RK)

$$
\begin{aligned}
\operatorname{Res} W_{i, j, k}^{(r)}= & \left.\frac{1}{\mu_{i, j, k}} \sum_{l=1}^{6}(\tilde{F}, \tilde{G}, \tilde{H})_{i, j, k, l}\right) \cdot \vec{n}_{i, j, k, l}^{0} \Delta S_{i, j, k, l} \\
W_{i, j, k}^{(0)}= & W_{i, j, k}^{n} \\
W_{i, j, k}^{r+1}= & W_{i, j, k}^{(0)}-\alpha_{r} \Delta t \operatorname{Res} W_{i, j, k}^{(r)}+A D\left(W_{i, j, k}^{n}\right), r=0,1,2 \\
W_{i, j, k}^{n+1}= & W_{i, j, k}^{(3)} \\
& \alpha_{0,1}^{n+1}=0.5, \alpha_{2}=1
\end{aligned}
$$

Each scheme was extended to include Jameson's artificial dissipation to improve the stability of the method.

$$
\begin{aligned}
A D\left(W_{i, j, k}^{n}\right)= & C_{1} \psi_{1}\left(W_{i-1, j, k}^{n}-2 W_{i, j, k}^{n}+W_{i+1, j, k}^{n}\right)+C_{2} \psi_{2}\left(W_{i, j-1, k}^{n}-2 W_{i, j, k}^{n}+W_{i, j+1, k}^{n}\right)+ \\
& +C_{3} \psi_{3}\left(W_{i, j, k-1}^{n}-2 W_{i, j, k}^{n}+W_{i, j, k+1}^{n}\right)
\end{aligned}
$$

where

$$
\psi_{1}=\frac{\left|p_{i-1, j, k}^{n}-2 p_{i, j}^{n}+p_{i+1, j, k}^{n}\right|}{\left|p_{i-1, j, k}^{n}\right|+\left|p_{i, j, k}^{n}\right|+\left|p_{i+1, j, k}^{n}\right|}, \psi_{2}=\frac{\left|p_{i, j-1, k}^{n}-2 p_{i, j, k}^{n}+p_{i, j+1, k}^{n}\right|}{\left|p_{i, j-1, k}^{n}\right|+\left|p_{i, j, k}^{n}\right|+\left|p_{i, j+1, k}^{n}\right|}, \psi_{3}=\frac{\left|p_{i, j, k-1}^{n}-2 p_{i, j, k}^{n}+p_{i, j, k+1}^{n}\right|}{\left|p_{i, j, k-1}^{n}\right|+\left|p_{i, j, k}^{n}\right|+\left|p_{i, j, k+1}^{n}\right|}
$$

The convergence to the steady state is followed by $\log L_{2}$ residual defined by

$$
\text { Res }=\sqrt{\frac{1}{N} \sum_{k}\left(\frac{W_{k}^{n+1}-W_{k}^{n}}{\Delta t}\right)}
$$

where $N$ is a number of all cells in the computational domain.

\section{FORMULATION OF THE PROBLEMS}

For an observation of effects of mentioned upstream boundary conditions on the numerical solution, we assumed a test case GAMM channel and its 3D modification (here called SWEPT WING), where a middle part of the lower wall represents the upper half of a swept wing). Their outlines are presented in Fig. 1, 2. 


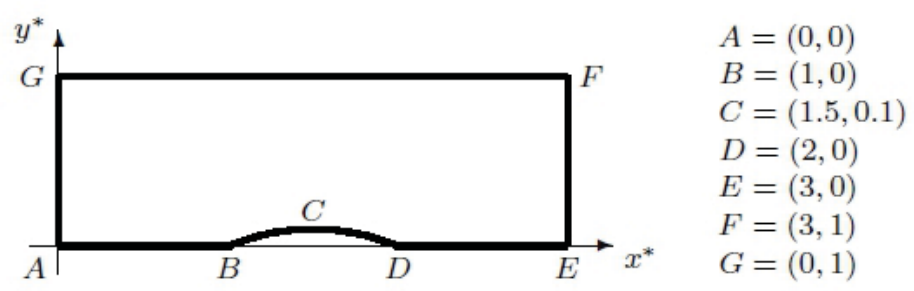

Fiaure 1: test case GAMM channel

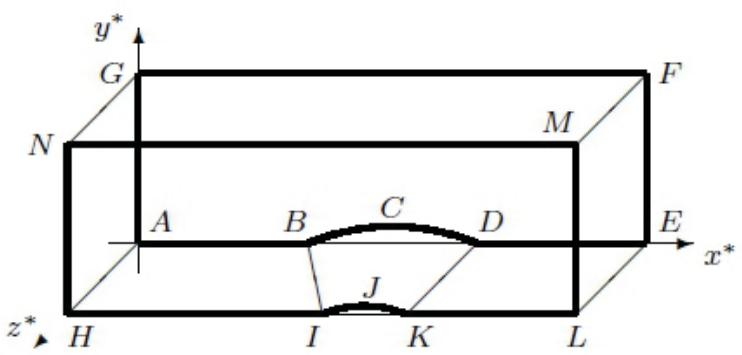

$$
\begin{array}{ll}
A=(0,0,0) & H=(0,0,1) \\
B=(1,0,0) & I=(1.5,0,1) \\
C=(1.5,0.1,0) & J=(1.75,0.04,1) \\
D=(2,0,0) & K=(2,0,1) \\
E=(3,0,0) & L=(3,0,1) \\
F=(3,1,0) & M=(3,1,1) \\
G=(0,1,0) & N=(0,1,1)
\end{array}
$$

Figure 2: test case SWEPT WING

\subsection{BOUNDARY CONDITIONS}

On the inlet we considered two types of inlet boundary conditions (IBC):

$\mathcal{A} \rho_{1}=1, u_{1}=M_{1}, v_{1}=0, w_{1}=0, p_{1}$ was extrapolated from the flow field and $e_{1}$ was calculated using the equation of state.

$\mathcal{B} \rho_{1}=1, u_{1}=M_{1}, v_{1}=0, w_{1}=0, p_{1}=1 / \kappa=0.714$ and $e_{1}$ was calculated using the equation of state.

On the outlet we prescribed only pressure $p_{2}=p_{1}$ and the other components were extrapolated from the flow field.

The velocity components on a solid wall were prescribed so that the normal velocity vectors equals to zero. This condition was implemented by using virtual cells. Such cells adjoin from outside on the boundary cells and we prescribed values of unknowns inside of them to obtain the desired effect.

Initial conditions were prescribed to comply with the inlet conditions.

\section{NumERICAL RESULTS}

For a 2D numerical simulation of flows of an inviscid compressible fluid in the GAMM channel, the authors applied both numerical schemes (LW and RK) and the boundary conditions IBC $\mathcal{A}, \mathcal{B}$ on a structured non-orthogonal grid with $240 \times 50$ cells. When the boundary condition IBC $\mathcal{A}$ was used - Fig. 3 (LW) and 5 (RK), maximums of Mach number on the lower wall $\left(M_{\max }=1.42\right.$ and 1.39 respectively) were larger than in the case of the IBC $\mathcal{B}\left(M_{\max }=1.35\right.$ and 1.34 respectively) - Fig. 4 (LW) and 6 (RK). But in the literature, the maximum of Mach number equals 1.37. Another way, there is a good or very good mutual agreement and similarly with J. Fürst results received by WLSQR scheme on two different computational grids with $150 \times 50\left(M_{\max }=1.29\right)$ and $300 \times 100$ cells $\left(M_{\max }=1.32\right)$ respectively, Fig. 7 . The dissimilarities of Mach number maximum $M_{\max }$ can be caused by used grids and numerical schemes.

In the case of a 3D inviscid compressible flow around the SWEPT WING, RK scheme and boundary condition IBC $\mathcal{A}$ on a structured non-orthogonal grid with $110 \times 30 \times 10$ cells were used. The results (Fig. 8) were compared with J. Holman results obtained by MUSCL (Fig. 9) and WLSQR scheme (Fig. 10) and in this case a good mutual agreement was found as well. It should be used a fine grid for a better detection of a Zierep singularity. 

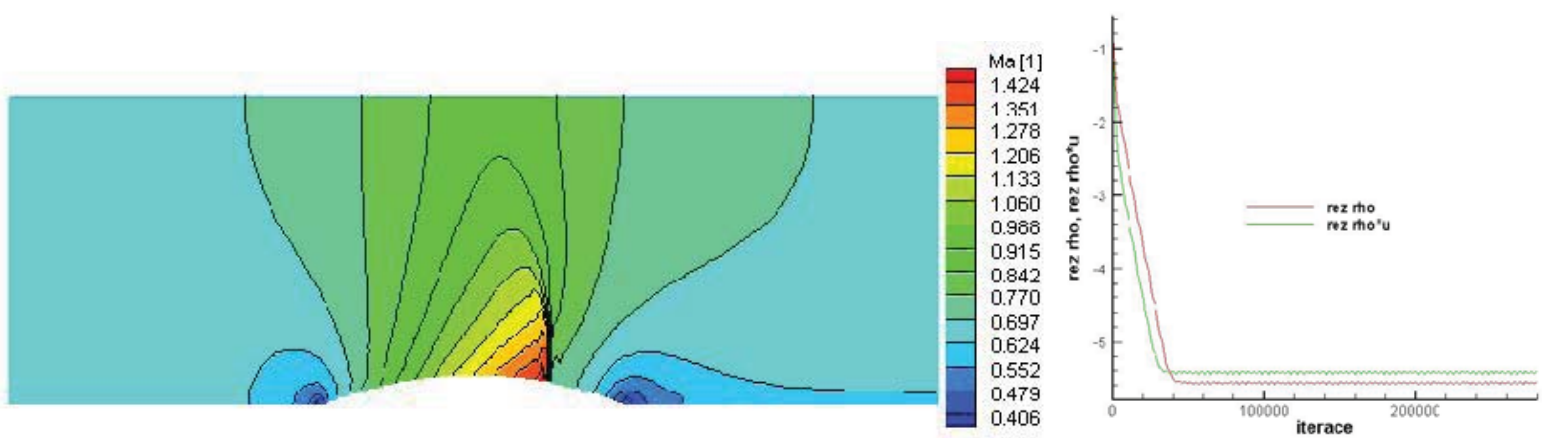

Figure 3: Inviscid compressible flow in the GAMM channel: Mach number isolines (left) and $\mathrm{L}_{2}$ logarithmic residuals (right) at $\mathrm{M}_{1}=0.675-\mathrm{LW}$ scheme, IBC $\mathcal{A}\left[\mathrm{M}_{\max }=1.42\right]$, a structured nonorthogonal grid with $240 \times 50$ cells.
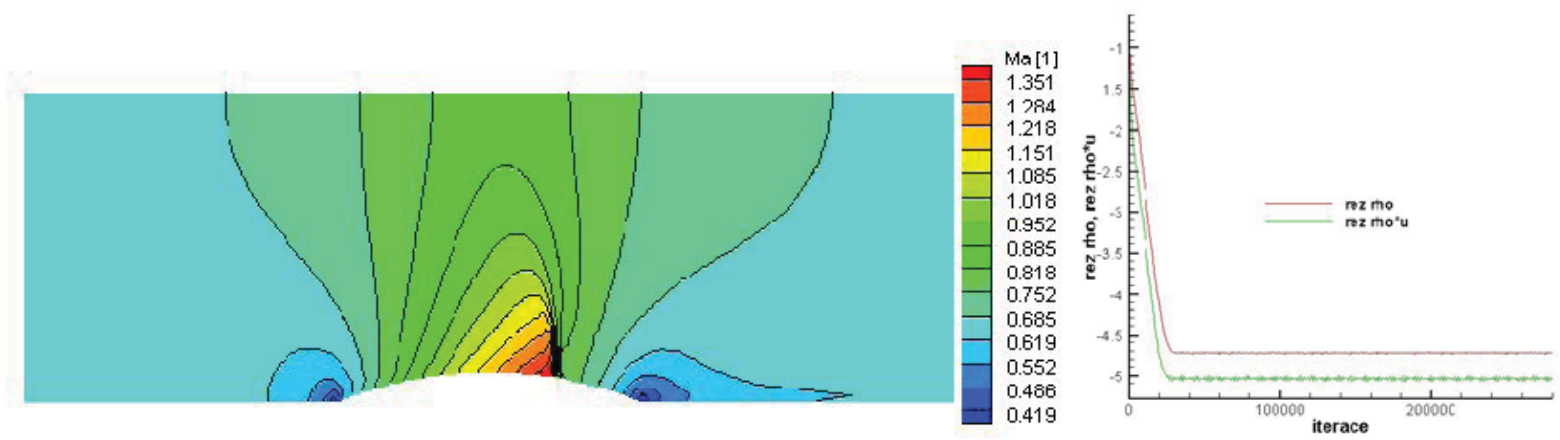

Figure 4: Inviscid compressible flow in the GAMM channel: Mach number isolines (left) and $\mathrm{L}_{2}$ logarithmic residuals (right) at $\mathrm{M}_{1}=0.675-\mathrm{LW}$ scheme, IBC $\mathcal{B}\left[\mathrm{M}_{\max }=1.35\right]$, a structured nonorthogonal grid with $240 \times 50$ cells.
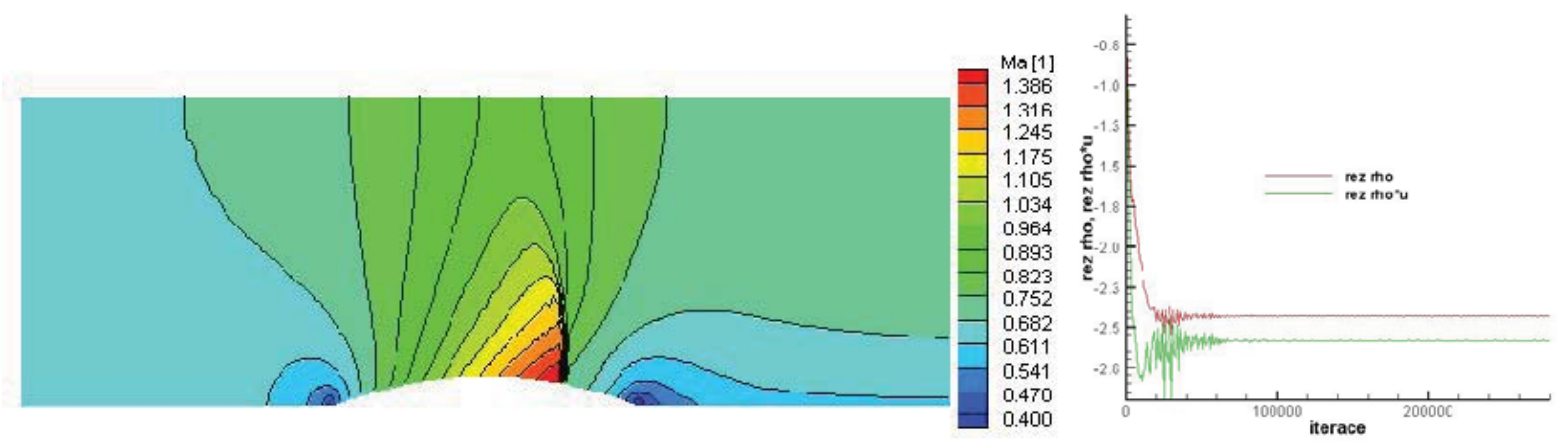

Figure 5: Inviscid compressible flow in the GAMM channel: Mach number isolines (left) and $\mathrm{L}_{2}$ logarithmic residuals (right) at $\mathrm{M}_{1}=0.675-\mathrm{RK}$ scheme, IBC $\mathcal{A}$ [ $\left.\mathrm{M}_{\max }=1.39\right]$, a structured nonorthogonal grid with $240 \times 50$ cells.
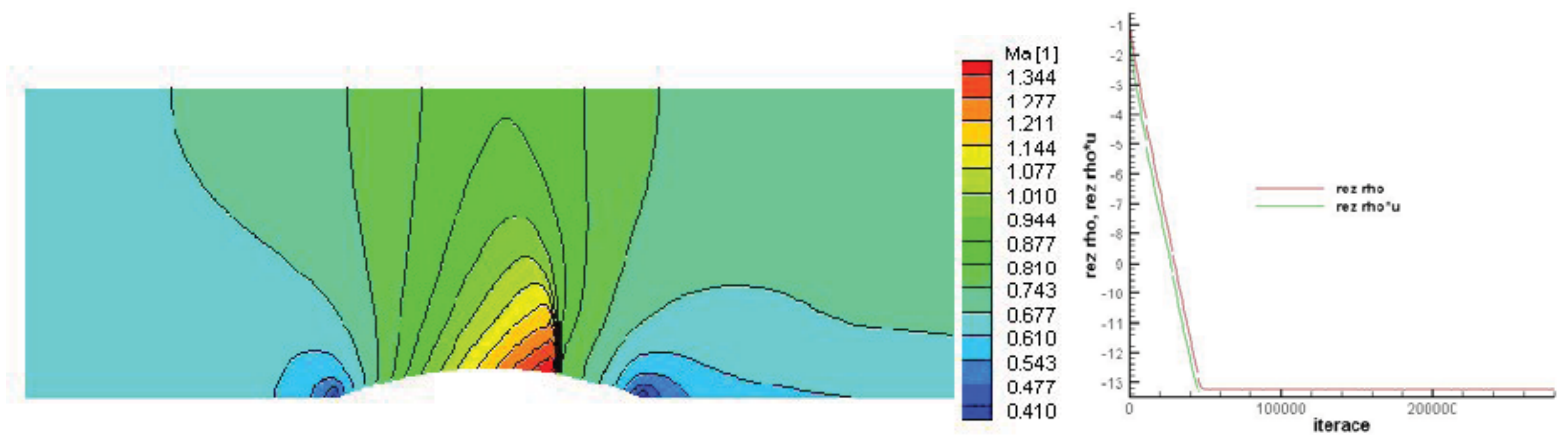

Figure 6: Inviscid compressible flow in the GAMM channel: Mach number isolines (left) and $\mathrm{L}_{2}$ logarithmic residuals (right) at $\mathrm{M}_{1}=0.675-\mathrm{RK}$ scheme, IBC $\mathcal{B}\left[\mathrm{M}_{\max }=1.34\right]$, a structured nonorthogonal grid with $240 \times 50$ cells. 

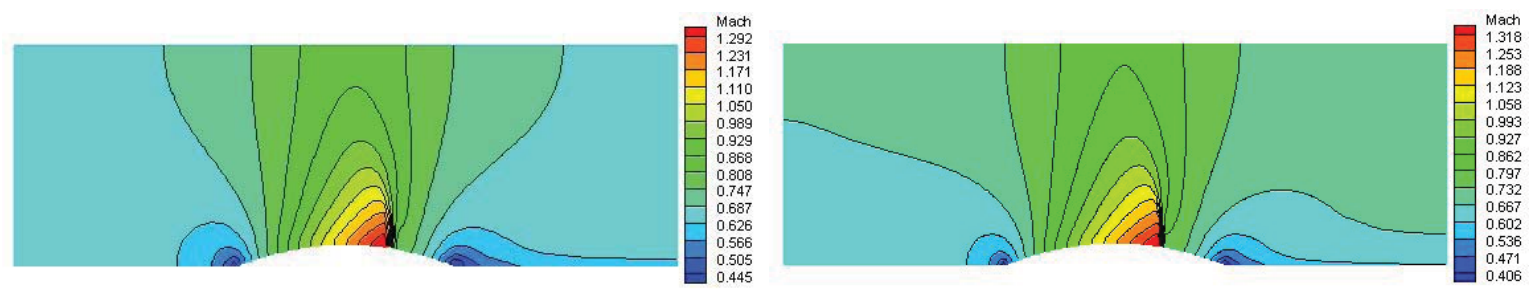

Figure 7: Inviscid compressible flow in the GAMM channel: Mach number isolines at $\mathrm{M}_{1}=\mathbf{0 . 6 7 5}$ - WLSQR scheme by J. Fürst [5], IBC $\mathcal{A}$, a structured non-orthogonal grid with $150 \times 50$ cells $\left[\mathrm{M}_{\max }=1.29\right.$ (left) $]$ and with $300 \times 100$ cells $\left[\mathrm{M}_{\max }=1.32\right.$ (right)].
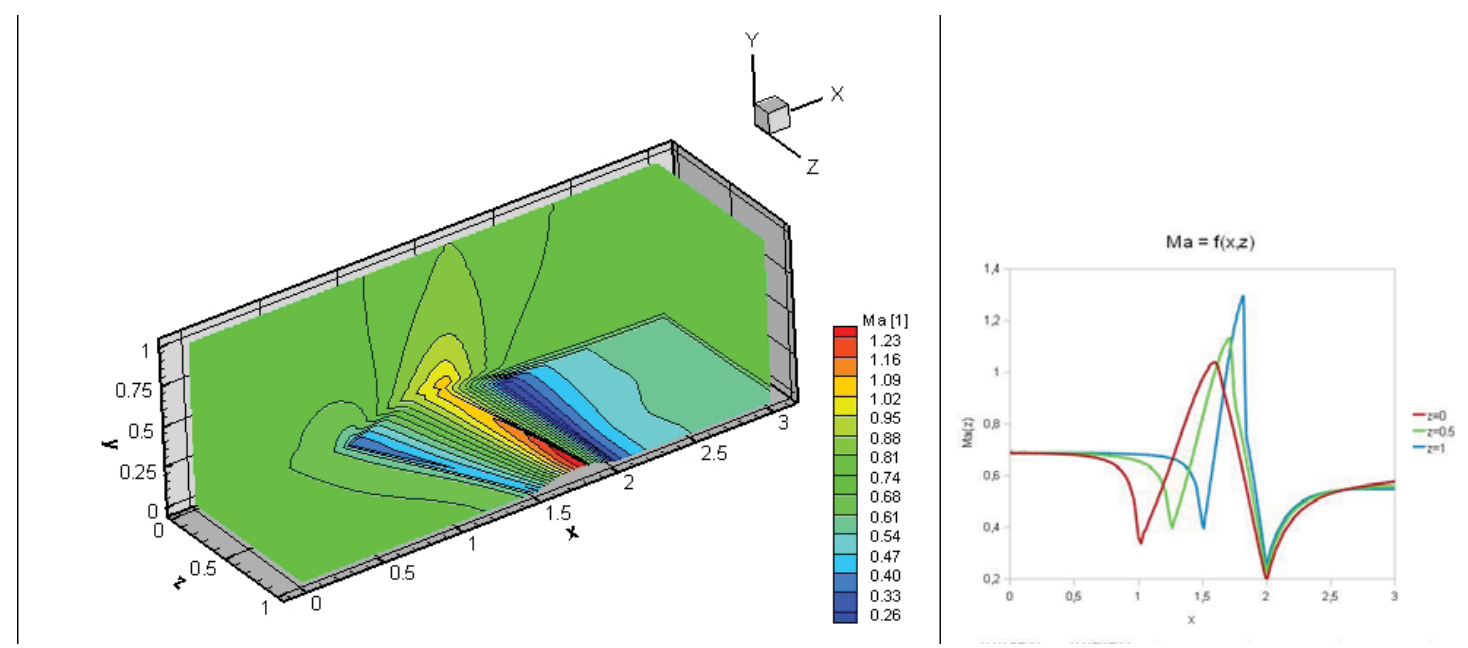

Figure 8: Inviscid compressible flow around the SWEPT WING in the channel: Mach number isolines at $\mathrm{M}_{1}=0.675$ (left) and distributions of Mach number on the lower wall in the slides (right) - RK scheme, IBC $\mathcal{A}$, a structured non-orthogonal grid with $110 \times 30 \times 10$ cells.
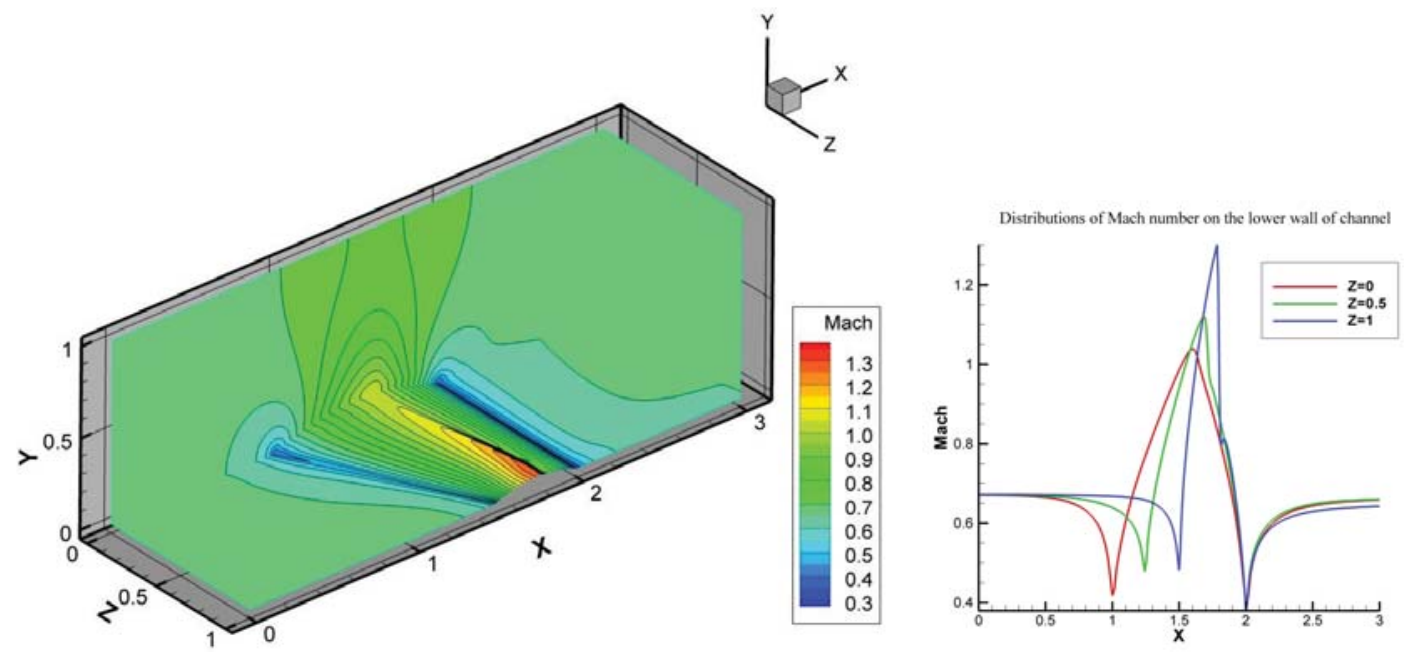

Figure 9: Inviscid compressible flow around the SWEPT WING in the channel: Mach number isolines at $\mathrm{M}_{1}=0.675$ (left) and distributions of Mach number on the lower wall in the slides (right) - MUSCL scheme by J. Holman [3], IBC $\mathcal{A}$, a structured non-orthogonal grid with $180 \times 35 \times 35$ cells. 

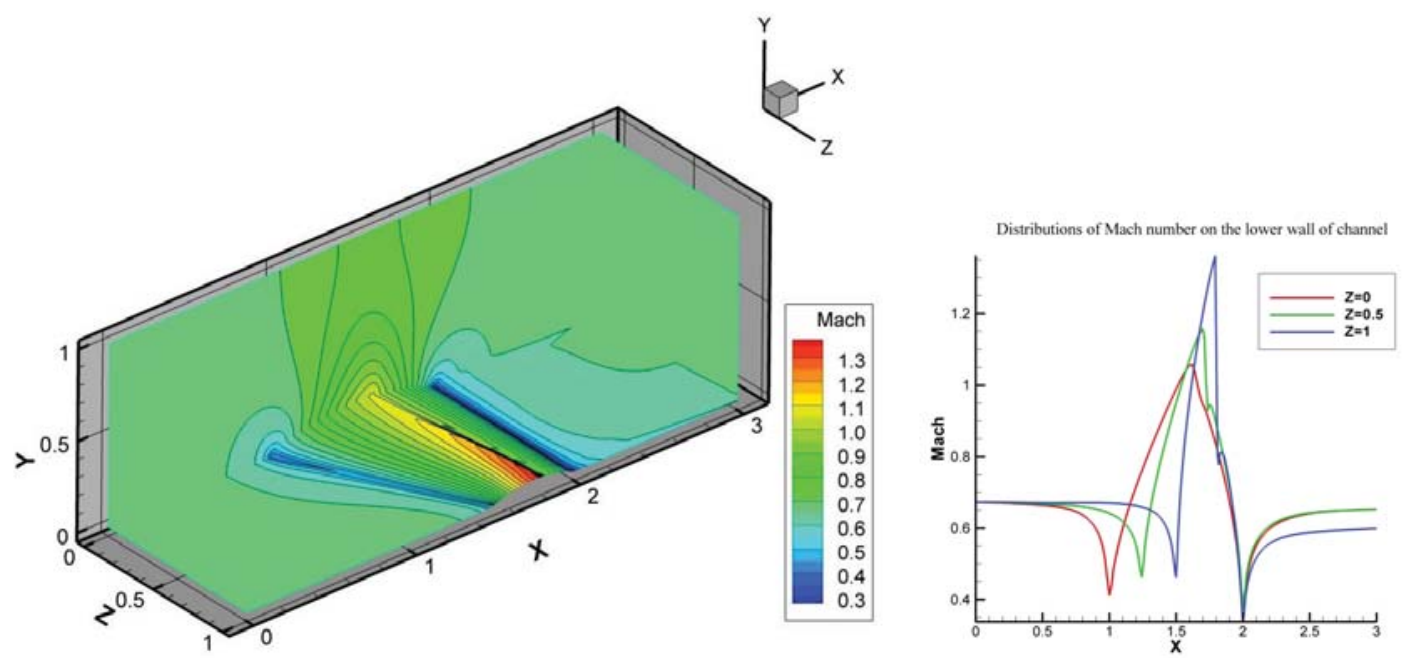

Figure 10: Inviscid compressible flow around the SWEPT WING in the channel: Mach number isolines at $\mathrm{M}_{1}=0.675$ (left) and distributions of Mach number on the lower wall in the slides (right) - WLSQR scheme by J. Holman [3], IBC $\mathcal{A}$, a structured non-orthogonal grid with $180 \times 35 \times 35$ cells.

\section{Conclusions}

According to the obtained results, it was found out that it is possible to prescribe on the inlet all the variables and the different values of Mach number maximum on the lower wall that occured is able to explain by the used numerical schemes and computational grids. Only for IBC $\mathcal{B}$ maximal Mach number seems to be in final solution slightly lower but all results are qualitatively the same.

\section{ACKNOWLEDGMENTS}

The work was partly supported by the Research Plan VZ MSM 6840770010 and by the grant projects GA AS CR IAA 20076080, GA CR P101/10/1329 and SGS 10/243/OHK2/3T/12.

\section{REFERENCES}

[1] Dvořák R.: Transonic Flows, Academia, Prague, 1986 (in Czech).

[2] Dvořák R., Kozel K.: Mathematical modelling in aerodynamics, CTU in Prague, Prague, 1996.

[3] Holman J., Fürst J.: Comparision of High Order Methods for Transonic Inviscid Flows, Proceedings: Colloquium Fluid Dynamics 2008, Institute of Thermomechanics, AS CR, v. v. i., Prague, 2008, pp. 11-12.

[4] Huml J., Fürst J., Kozel K., Př́hoda J.: Numerical Solution of Subsonic and Transonic Flows in a Channel, Proceedings: Topical Problems of Fluid Dynamics 2009, Institute of Thermodynamics, AS CR, v. v. i., Prague, 2009, pp. 45-48 (in Czech).

[5] Huml J., Holman J., Fürst J., Kozel K.: Numerical Solution of 3D Inviscid Flows in a Channel, Proceedings: Topical Problems of Fluid Dynamics 2010, Institute of Thermomechanics, AS CR, v. v. i., Prague, 2010, pp. 73-76. 